\title{
HOMOMORPHISMS OF ERGODIC GROUP ACTIONS AND CONJUGACY OF SKEW PRODUCT ACTIONS
}

\author{
EDGAR N. REYES \\ Department of Mathematics \\ Southeastern Louisiana University \\ Hammond, Louisiana 70402 \\ (Received October 19, 1994 and in revised form June 1, 1995)

\begin{abstract}
Let $G$ be a locally compact group acting ergodically on $X$. We discuss relationships between homomorphisms on the measured groupoid $X \times G$, conjugacy of skew product extensions, and similarity of measured groupoids. To do this, we describe the structure of homomorphisms on $X \times G$ whose restriction to an extension given by a skew product action is the trivial homomorphism.
\end{abstract}

KEY WORDS AND PHRASES. Homomorphism between ergodic group actions, groupoids, Mackey dense range, cocycles, skew products.

1991 AMS SUBJECT CLASSIFICATION CODES. 20L13, 22D40, $28 \mathrm{D} 15$.

1. INTRODUCTION. Ergodic group actions is one subfamily of the categorical generalization of locally compact groups as suggested by Mackey in [3]. This generalization include measured equivalence relations, measured foliations, and measured ergodic groupoids amongst others.

A concept for groups that extends to this category and in particular to ergodic group actions is the homomorphism. This paper discusses relationships between homomorphisms of ergodic group actions, their restrictions, conjugacy of skew product actions, and similarity of measured groupoids. These relationships provide an answer to the following problem about locally compact groups.

If $\phi: G \rightarrow H$ and $\psi: G \rightarrow K$ are continuous homomorphisms whose ranges are dense in $H$ and $K$, respectively, does there exists an isomorphism $\eta: H \rightarrow K$ satisfying $\psi=\eta \circ \phi$ ? If so, $H$ and $K$ are conjugate $G$-spaces where the action of $G$ on $H$ is given by $h \cdot g=h \phi(g)$. We will investigate the extent to which this problem generalizes to ergodic group actions. This paper will extend some of the results obtained by Fabec in $[1,2]$.

Before stating the main result, which is the theorem at the end of this section, we will briefly review notions of group actions and homomorphisms of measured groupoids. The proof of the main result will be given in the second section. In the third section, the theorem will be applied to find relationships between conjugacy of skew product extensions of an ergodic group action and similarity of measured groupoids. In particular, the above problem is answered there. 
Let $G$ be a second countable, locally compact group. Suppose $\mu$ is a $\sigma$-finite measure on a standard Borel space $X$. Then $X$ is a $G$-space provided there exists a Borel mapping $(x, g) \mapsto x \cdot g$ from $X \times G$ to $X$ satisfying

(1) $x \cdot e=x$ for all $x$,

(2) $x \cdot g_{1} \cdot g_{2}=x \cdot g_{1} g_{2}$ for all $x, g_{1}, g_{2}$, and

(3) $\mu(E \cdot g)=0$ if and only if $\mu(E)=0$ for each $g$ and Borel subset $E$.

Two $G$-spaces $\left(X_{1}, \mu_{1}\right)$ and $\left(X_{2}, \mu_{2}\right)$ are isomorphic if there exists a Borel isomorphism $\Phi: X_{1} \rightarrow X_{2}$ such that $\Phi(x \cdot g)=\Phi(x) \cdot g \mu_{1}$-a.e. $x$ for each $g$ and $\Phi_{*} \mu_{1} \equiv \mu_{1} \circ \Phi^{-1} \sim \mu_{2}$ i.e. $\Phi_{*} \mu_{1}$ and $\mu_{2}$ belong to the same measure class.

If $(Y, \nu)$ is a $G$-space then a Borel map $p: X \rightarrow Y$ is called an extension if $p(x \cdot g)=p(x) \cdot g$ for all $(x, g)$ and $p_{*} \mu \sim \nu$. Two extensions $p_{1}:\left(X_{1}, \mu_{1}\right) \rightarrow(Y, \nu)$ and $p_{2}:\left(X_{2}, \mu_{2}\right) \rightarrow(Y, \nu)$ are isomorphic over $Y$ if there exists a $G$-space isomorphism $\Phi$ from $X_{1}$ to $X_{2}$ such that $p_{2} \circ \Phi(x)=p_{1}(x)$ a.e. $x$.

Let $H$ be a complete separable metric group. A cocycle on the $G$-space $(X, \mu)$ with values in $H$ is a Borel mapping $\phi: X \times G \rightarrow H$ satisfying $\phi\left(x, g_{1} g_{2}\right)=\phi\left(x, g_{1}\right) \phi\left(x \cdot g_{1}, g_{2}\right)$ for all $x, g_{1}, g_{2}$. If one defines $(x, h) \cdot g=(x \cdot g, h \phi(x, g))$ then $\left(X \times H, \mu \times m_{H}\right)$ becomes a $G$-space where $m_{H}$ is a Haar measure on $H$. This $G$ space is denoted by $\left(X \times_{\phi} H, \mu \times m_{H}\right)$ and is called the skew product action defined by the cocycle $\phi$.

The measure space $\left(X \times G, \mu \times m_{G}\right)$ is called the measured groupoid defined by the action of $G$ on $(X, \mu)$. There is a partially defined multiplication on the groupoid, namely,

$(x, g)\left(x \cdot g, g_{1}\right)=\left(x, g g_{1}\right)$. Also, $(x, g)^{-1}=\left(x \cdot g, g^{-1}\right)$ defines an inverse operation in the groupoid. Given $z_{1}, z_{2}, z_{3}, z_{4} \in X \times G$, one can directly verify that

$$
\text { if } z_{1} z_{2}=z_{3} \text { then } z_{1}^{-1} z_{3} \text { is well-defined and } z_{2}=z_{1}^{-1} z_{3} \text {. }
$$

A homomorphism $h: X \times G \rightarrow Y \times H$ between measured groupoids or group actions is a pair of Borel maps $h=(p, \phi)$ where $p: X \rightarrow Y$ and $\phi: X \times G \rightarrow H$ satisfy

(4) $\phi$ is a cocycle,

(5) $p(x \cdot g)=p(x) \cdot \phi(x, g)$ for all $(x, g)$, and

(6) $\mu\left(p^{-1}(E)\right)=0$ if and only if $\nu(E)=0$ whenever $E$ is an invariant analytic subset of $Y$.

Statements (4) and (5) are necessary and sufficient conditions for $h: X \times G \rightarrow Y \times H$ to define a multiplicative mapping between groupoids. An action of $G$ is ergodic if $E$ is a null or conull Borel subset whenever $E \cdot g=E$ for all $g$. In case $(X, \mu)$ is an ergodic $G$-space and $(Y, \nu)$ is an ergodic $H$-space, one says $h$ is a homomorphism between ergodic group actions. In this case, as pointed out by Ramsay in [5, page 394], statement (6) is equivalent to the condition: $p^{-1}(E)$ is null whenever $E$ is an invariant analytic null subset of $Y$.

Two homomorphisms $h$ and $h^{\prime}$ from $X \times G$ to $Y \times H$ are cohomologous or similar if there is a Borel mapping $A: X \rightarrow Y \times H$ satisfying $A(x) h(x, g)=h^{\prime}(x, g) A(x \cdot g)$ a.e. $(x, g)$. This will be denoted by $h \sim h^{\prime}$. The idea of isomorphic groups extends to the notion of similar measured groupoids. Let $i d$ be the identity mapping. If there exist homomorphisms $J_{1}: X \times G \rightarrow Y \times H$ and $J_{2}: Y \times H \rightarrow X \times G$ for which $J_{1} \circ J_{2} \sim i d$ and $J_{2} \circ J_{1} \sim i d$, then $X \times G$ and $Y \times H$ are called similar measured groupoids. Also, a homomorphism $h: X \times G \rightarrow Y \times H$ is said to be trivial 
if there is a Borel mapping $A: X \rightarrow Y \times H$ satisfying

$$
A(x) h(x, g)=A(x \cdot g) \text { a.e. }(x, g)
$$

A homomorphism $h=(p, \phi): X \times G \rightarrow Y \times H$ between ergodic group actions is said to have Mackey dense range if $(x, h) \mapsto p(x) h^{-1}$ provides an ergodic decomposition of the skew product $G$-space $X \times_{\phi} H$. In particular, a cocycle $\phi$ has Mackey dense range in the locally compact group $H$ if the skew product action is ergodic. An ergodic decomposition satisfies a universal property. Namely, if $f$ is a Borel function on $X \times_{\phi} H$ with values in a countably separated Borel space and $f$ is essentially invariant, i.e. $f(x \cdot g, h \phi(x, g))=f(x, h)$ a.e. $(x, h, g)$, then there exists an essentially unique Borel function $F$ defined on $Y$ such that $F\left(p(x) \cdot h^{-1}\right)=f(x, h)$ a.e. $(x, h)$.

One can also restrict homomorphisms between measured groupoids. Indeed, if $p_{0}: X \rightarrow Y$ is an extension of $G$-spaces, the homomorphism given by $i(x, g)=\left(p_{0}(x), g\right)$ from $X \times G$ into $Y \times G$ is called an inclusion homomorphism. If $h=(p, \phi)$ is a homomorphism of $Y \times G$ into $W \times H$, the composition $h \circ i$ is called the restriction of $h$ to $X \times G$ and will be denoted by $\left.h\right|_{X \times G}$. So $\left.h\right|_{X \times G}(x, g)=\left(p\left(p_{0}(x)\right), \phi\left(p_{0}(x), g\right)\right)$ for all $(x, g)$. The main result of this paper is the following theorem and it is an extension of Proposition 2.1 in Fabec [2].

THEOREM Let $h=(p, \phi): X \times G \rightarrow Y \times H$ be a homomorphism with Mackey dense range and let $b=(q, \psi): X \times G \rightarrow Z \times K$ be a homomorphism between ergodic group actions. Then $b \sim a \circ h$ for some homomorphism $a: Y \times H \rightarrow Z \times K$ if and only if $\left.b\right|_{X \times_{\phi} H \times G}$ is trivial.

2. PROOF OF THEOREM. To organize and divide the proof, we will begin with two lemmas. It will be shown that by changing a trivial homomorphism slightly one can change the 'almost everywhere' condition in (1.2) to one that will hold 'for all'.

LEMMA 2.1 Let $W$ be a $G$-space and let $\psi: W \times G \rightarrow K$ be a cocycle. Suppose $\eta: W \rightarrow K$ is a Borel mapping such that $\eta(w) \psi(w, g)=\eta(w \cdot g)$ a.e. $(w, g)$. Then there exist a cocycle $\psi_{0}: W \times G \rightarrow K$, a Borel mapping $A_{2}: W \rightarrow K$, and a conull, Borel subset $W_{0}$ of $W$ with $W_{0} \cdot G$ Borel satisfying

(a) $A_{2}(w) \psi_{0}(w, g)=A_{2}(w \cdot g)$ for all $(w, g)$,

(b) $\psi_{0}(w, g)=\psi(w, g)$ for all $w \in W_{0} \cdot G$ and $g \in G$, and

(c) $A_{2}(w)=\eta(w)$ a.e. $w$.

PROOF. Let $J=\left\{(w, g): \eta(w)=\eta(w \cdot g) \psi(w, g)^{-1}\right\}$. If $\left(w, g_{1}\right)$ and $\left(w \cdot g_{1}, g_{2}\right)$ are in $J$ then $\eta\left(w \cdot g_{1} g_{2}\right) \psi\left(w, g_{1} g_{2}\right)^{-1}=\eta\left(w \cdot g_{1} g_{2}\right) \psi\left(w \cdot g_{1}, g_{2}\right)^{-1} \psi\left(w, g_{1}\right)^{-1}=\eta\left(w \cdot g_{1}\right) \psi\left(w, g_{1}\right)^{-1}=\eta(w)$ and so $\left(w, g_{1} g_{2}\right) \in J$. Since $J$ is a conull, multiplicatively closed Borel subset of the groupoid $W \times G$, then by Lemma A.4 in Fabec [2] and Lemma 5.2 in Ramsay [4] there is a Borel, conull subset $W_{0}$ of $W$ such that

(i) if $w, w \cdot g \in W_{0}$ then $(w, g) \in J$,

(ii) $W_{0} \cdot G$ is a Borel subset, and

(iii) there exists a Borel mapping $\theta: W_{0} \cdot G \rightarrow G$ such that $\theta(w)=e$ if $w \in W_{0}$, and $w \cdot \theta(w) \in W_{0}$ for all $w \in W_{0} \cdot G$. 
If $w \in W_{0} \cdot G$ then define $A_{2}(w)=\eta(w \cdot \theta(w)) \psi(w, \theta(w))^{-1}$, otherwise set $A_{2}(w)=e$.

Let $\psi_{0}: W \times G \rightarrow K$ be the cocycle defined by $\psi_{0}(w, g)=\psi(w, g)$ if $w \in W_{0} \cdot G$, and if $w \notin W_{0} \cdot G$ set $\psi_{0}(w, g)=e$. If $w \in W_{0} \cdot G, g \in G$ then $\left(w \cdot \theta(w), \theta(w)^{-1} g \theta(w \cdot g)\right) \in J$, and cosequently, $A_{2}(w \cdot g) \psi_{0}(w, g)^{-1}=\eta(w \cdot g \theta(w \cdot g)) \psi(w \cdot g, \theta(w \cdot g))^{-1} \psi(w, g)^{-1}=$ $\eta(w \cdot g \theta(w \cdot g)) \psi(w, g \theta(w \cdot g))^{-1}=\eta(w \theta(w)) \psi\left(w \cdot \theta(w), \theta(w)^{-1} g \theta(w \cdot g)\right) \psi(w, g \theta(w \cdot g))^{-1}=$ $\eta(w \theta(w)) \psi\left(w \cdot \theta(w), \theta(w)^{-1}\right)=A_{2}(w)$. Hence, $A_{2}(w \cdot g) \psi_{0}(w, g)^{-1}=A_{2}(w)$ for all $(w, g)$.

LEMMA 2.2 Let $b=(q, \psi): W \times G \rightarrow Z \times K$ be a trivial homomorphism between measured groupoids. Then there exist Borel mappings $b_{0}=\left(q_{0}, \psi_{0}\right): W \times G \rightarrow Z \times K$, $A_{0}=\left(A_{1}, A_{2}\right): W \rightarrow Z \times K$, and a conull,Borel subset $W_{0}$ of $W$ with $W_{0} \cdot G$ Borel satisfying

(a) $\psi_{0}: W \times G \rightarrow K$ is a cocycle where $\psi_{0}(w, g)=\psi(w, g)$ for all $w \in W_{0} \cdot G$ and $g \in G$,

(b) $q(w)=q_{0}(w)$ a.e $w$, and

(c) $A_{0}(w) b_{0}(w, g)=A_{0}(w \cdot g)$ for all $(w, g)$.

PROOF. Let $A=(\xi, \eta): W \rightarrow Z \times K$ be a Borel mapping satisfying $(\xi(w), \eta(w))(q(w), \psi(w, g))=(\xi(w \cdot g), \eta(w \cdot g))$ a.e. $(w, g)$. By Lemma 2.1 we can choose a cocycle $\psi_{0}: W \times G \rightarrow K$, a Borel mapping $A_{2}: W \rightarrow K$, and a conull, Borel subset $W_{0}$ with $W_{0} \cdot G$ Borel such that (a) - (c) of Lemma 2.1 holds. Let $J=\{(w, g): \xi(w)=\xi(w \cdot g)\}$. Since $J$ is a conull, multiplicatively closed Borel subset of the groupoid $W \times G$ then by Lemma A.4 in Fabec [2] and Lemma 5.2 in Ramsay [4] there exists a conull, Borel subset $W_{1}$ of $W$ such that

(i) if $w, w \cdot g \in W_{1}$ then $(w, g) \in J$,

(ii) $W_{1} \cdot G$ is a Borel subset, and

(iii) there exists a Borel mapping $\theta: W_{1} \cdot G \rightarrow G$ where $\theta(w)=e$ for $w \in W_{1}$, and $w \cdot \theta(w) \in W_{1}$ for all $w \in W_{1} \cdot G$.

Let $A_{1}: W \rightarrow Z$ be the Borel mapping given by $A_{1}(w)=\xi(w \cdot \theta(w))$ if $w \in W_{1} \cdot G$ and if $w \notin W_{1} \cdot G$ set $A_{1}(w)=z_{0}$ where $z_{0}$ is some fixed element of $Z$ independent of $w$. If $w \in W_{1} \cdot G$ then $\left(w \theta(w), \theta(w)^{-1} g \theta(w \cdot g)\right) \in J$ and so $A_{1}(w \cdot g)=\xi(w \cdot g \theta(w \cdot g))=\xi(w \theta(w))=A_{1}(w)$. Thus, $A_{1}(w \cdot g)=A_{1}(w)$ for all $(w, g)$.

Define a Borel map $q_{0}: W \rightarrow Z$ by letting $q_{0}(w)=A_{1}(w) A_{2}(w)$. Since $A_{2}(w)=\eta(w)$ a.e. $w$ and $\xi(w) \cdot \eta(w)=q(w)$ a.e. $w$ then $q_{0}(w)=q(w)$ a.e. $w$. Set $b_{0}=\left(q_{0}, \psi_{0}\right)$ and $A_{0}=\left(A_{1}, A_{2}\right)$. Then $\left(A_{1}(w), A_{2}(w)\right)\left(q_{0}(w), \psi_{0}(w, g)\right)=\left(A_{1}(w \cdot g), A_{2}(w \cdot g)\right)$ for all $w, g$.

Turning to the proof of the Theorem, suppose $b \sim a \circ h$. Since 'cohomologous' defines an equivalence relation, one can assume $b=a \circ h$. If $A(x, h)=a\left(p(x) h^{-1}, h\right)$ then $A(x, h) b(x, g)=$ $a\left(p(x) h^{-1}, h\right) a(p(x), \phi(x, g))=a\left(p(x) h^{-1}, h \phi(x, g)\right)=a\left(p(x g) \phi(x, g)^{-1} h^{-1}, h \phi(x, g)\right)=$ $A(x g, h \phi(x, g))$. Thus, $A(x, h) b(x, g)=A((x, h) \cdot g)$ for all $(x, h), g$ and $\left.b\right|_{X \times H \times G}$ is trivial.

Conversely, suppose $\left.b\right|_{X \times_{\phi} H \times G}: X \times_{\phi} H \times G \rightarrow Z \times K$ is trivial. According to Lemma 2.2, with $W=X \times_{\phi} H$, there are Borel mappings $b_{0}=\left(q_{0}, \psi_{0}\right): X \times_{\phi} H \times G \rightarrow Z \times K$ and $A_{0}=\left(A_{1}, A_{2}\right): X \times_{\phi} H \rightarrow Z \times K$ and conull Borel subsets $W_{0}, W_{2} \subseteq X \times_{\phi} H$, with $W_{0} \cdot G$ Borel for which

(a) $\psi_{0}$ is a cocycle and $\psi_{0}(x, h, g)=\psi(x, g)$ for all $(x, h) \in W_{0} \cdot G, g \in G$,

(b) $q(x)=q_{0}(x, h)$ for all $(x, h) \in W_{2}$, and

(c) $A_{0}(x, h) b_{0}(x, h, g)=A_{0}(x \cdot g, h \phi(x, g))$ for all $x, h, g$. 
Let $W_{3}=W_{2} \cap\left(W_{0} \cdot G\right)$. Then $b_{0}(x, h, g)=b(x, g)$ for all $(x, h) \in W_{3}, g \in G$.

Set $E=\left\{\left(x, h_{1}, h_{2}\right):\left(x, h_{1}\right),\left(x, h_{2}^{-1} h_{1}\right) \in W_{3}\right\}$. If $\left(x, h_{1}, h_{2}\right) \in E, g \in G$ then $A_{0}\left(x, h_{1}\right) b(x, g)=$ $A_{0}\left(x \cdot g, h_{1} \phi(x, g)\right)$ and $A_{0}\left(x, h_{2}^{-1} h_{1}\right) b(x, g)=A_{0}\left(x \cdot g, h_{2}^{-1} h_{1} \phi(x, g)\right)$. In particular, by (1.1), $A_{0}\left(x, h_{1}\right) A_{0}\left(x, h_{2}^{-1} h_{1}\right)^{-1}$ is a well-defined element of $Z \times K$ for all $\left(x, h_{1}, h_{2}\right) \in E$. Fix a point $z_{0}$ in $Z$ and define a Borel mapping $F: X \times_{\phi} H \times H \rightarrow Z \times K$ by

$$
F\left(x, h_{1}, h_{2}\right)= \begin{cases}A_{0}\left(x, h_{1}\right) A_{0}\left(x, h_{2}^{-1} h_{1}\right)^{-1} & \text { if }\left(x, h_{1}, h_{2}\right) \in E \\ \left(z_{0}, e\right) & \text { otherwise }\end{cases}
$$

Given $g \in G,\left(x, h_{1}, h_{2}\right) \in E$ such that $\left(x \cdot g, h_{1} \phi(x, g), h_{2}\right) \in E$, then $F\left(x \cdot g, h_{1} \phi(x, g), h_{2}\right)=$ $A_{0}\left(x \cdot g, h_{1} \phi(x, g)\right) A_{0}\left(x \cdot g, h_{2}^{-1} h_{1} \phi(x, g)\right)^{-1}=\left[A_{0}\left(x, h_{1}\right) b(x, g)\right]\left[A_{0}\left(x, h_{2}^{-1} h_{1}\right) b(x, g)\right]^{-1}=$ $A_{0}\left(x, h_{1}\right) A_{0}\left(x, h_{2}^{-1} h_{1}\right)^{-1}=F\left(x, h_{1}, h_{2}\right)$. Since $E$ is a conull, Borel subset and $\left(x, h_{1}\right) \mapsto p(x) h_{1}^{-1}$ is an ergodic decomposition, there exists a Borel mapping $a: Y \times H \rightarrow Z \times K$ satisfying $a\left(p(x) h_{1}^{-1}, h_{2}\right)=$ $A_{0}\left(x, h_{1}\right) A_{0}\left(x, h_{2}^{-1} h_{1}\right)^{-1}$ a.e. $\left(x, h_{1}, h_{2}\right)$. Moreover, $a\left(p(x) h^{-1}, h_{1} h_{2}\right)=A_{0}(x, h) A_{0}\left(x, h_{2}^{-1} h_{1}^{-1} h\right)^{-1}$ $=A_{0}(x, h) A_{0}\left(x, h_{1}^{-1} h\right)^{-1} A_{0}\left(x, h_{1}^{-1} h\right) A_{0}\left(x, h_{2}^{-1} h_{1}^{-1} h\right)^{-1}=a\left(p(x) h^{-1}, h_{1}\right) a\left(p(x) h^{-1} h_{1}, h_{2}\right)$ a.e. $\left(x, h, h_{1}, h_{2}\right)$, and hence, $a$ is almost multiplicative. By Proposition A.7 in Fabec [2], one can assume $a$ is strictly multiplicative. Since $a$ is given as a pair of Borel maps, there exist a Borel function $s: Y \rightarrow Z$ and a cocycle $\eta: Y \times H \rightarrow K$ which by definition satisfies

$$
\begin{aligned}
& \left(s\left(p(x) h_{1}^{-1}\right), \eta\left(p(x) h_{1}^{-1}, h_{2}\right)\right)= \\
& \quad=\left(A_{1}\left(x, h_{1}\right), A_{2}\left(x, h_{1}\right)\right)\left(A_{1}\left(x, h_{2}^{-1} h_{1}\right) \cdot A_{2}\left(x, h_{2}^{-1} h_{1}\right), A_{2}\left(x, h_{2}^{-1} h_{1}\right)^{-1}\right)
\end{aligned}
$$

for a.e. $\left(x, h_{1}, h_{2}\right)$. So $\eta\left(p(x) h_{1}^{-1}, h_{2}\right)=A_{2}\left(x, h_{1}\right) A_{2}\left(x, h_{2}^{-1} h_{1}\right)^{-1}$ a.e. $\left(x, h_{1}, h_{2}\right)$. Set $L(x, h)=$ $A_{2}(x, h)^{-1} \eta\left(p(x) h^{-1}, h\right)$. Then $L\left(x, h_{2}^{-1} h_{1}\right)=A_{2}\left(x, h_{2}^{-1} h_{1}\right)^{-1} \eta\left(p(x) h_{1}^{-1} h_{2}, h_{2}^{-1} h_{1}\right)=$ $A_{2}\left(x, h_{1}\right)^{-1} \eta\left(p(x) h_{1}^{-1}, h_{2}\right) \eta\left(p(x) h_{1}^{-1} h_{2}, h_{2}^{-1} h_{1}\right)=A_{2}\left(x, h_{1}\right)^{-1} \eta\left(p(x) h_{1}^{-1}, h_{1}\right)=L\left(x, h_{1}\right)$

a.e. $\left(x, h_{1}, h_{2}\right)$. Since $G$ is the only conull mutiplicatively closed subset of a group, $L\left(x, h_{2}^{-1} h_{1}\right)=$ $L\left(x, h_{1}\right)$ a.e. $h_{1}$, for each $h_{2}$, a.e. $x$. Thus, by Lemma A.3 in Fabec [2], there exists a Borel mapping $B: X \rightarrow K$ such that $B(x)=L(x, h)^{-1}$ a.e. $(x, h)$. It follows from the definition of $L(x, h)$ that

$$
A_{2}(x, h)=\eta\left(p(x) h^{-1}, h\right) B(x) \text { a.e. }(x, h)
$$

and so

$$
\begin{aligned}
A_{2}(x \cdot g, h \phi(x, g)) & =\eta\left(p(x \cdot g) \phi(x, g)^{-1} h^{-1}, h \phi(x, g)\right) B(x \cdot g) \\
& =\eta\left(p(x) h^{-1}, h\right) \eta(p(x), \phi(x, g)) B(x \cdot g) \\
& =A_{2}(x, h) B(x)^{-1} \eta(p(x), \phi(x, g)) B(x \cdot g)
\end{aligned}
$$

a.e. $(x, h)$ for each $g$. On the other hand, $A_{2}(x \cdot g, h \phi(x, g))=A_{2}(x, h) \psi_{0}(x, h, g)=A_{2}(x, h) \psi(x, g)$ a.e. $(x, h, g)$ by (a) and (c). Together with (2.3), one obtains

$$
\psi(x, g)=B(x)^{-1} \eta(p(x), \phi(x, g)) B(x \cdot g) \quad \text { a.e. }(x, g) .
$$

Since $s\left(p(x) h^{-1}\right)=A_{1}(x, h)$ a.e. $(x, h)$ by $(2.1)$ and $A_{1}(x, h) A_{2}(x, h)=q_{0}(x, h)$ for all $(x, h)$ by (c), then $q(x)=s\left(p(x) h^{-1}\right) A_{2}(x, h)$ a.e. $(x, h)$ by (b). Combining this with (2.2), one has $q(x)=s\left(p(x) h^{-1}\right) \eta\left(p(x) h^{-1}, h\right) B(x)=s(p(x)) B(x)$ a.e. $x$. 
Let $r: X \rightarrow Z$ be the Borel function given by $r(x)=s(p(x))$. So $q(x)=r(x) B(x)$ a.e. $x$ and $s(p(x)) \eta(p(x), \phi(x, g))=s(p(x) \phi(x, g))=s(p(x \cdot g))=r(x \cdot g)$. Thus, by (2.4), one obtains $(r(x), B(x))(q(x), \psi(x, g))=(s(p(x)), \eta(p(x), \phi(x, g)))(r(x \cdot g), B(x \cdot g))$ a.e. $(x, g)$.

Finally, we have to show $a=(s, \eta)$ satisfies $(6)$ of the definition of a homomorphism. Let $Z_{0}$ be an invariant analytic null subset of $Z$. So $\left\{x: s(p(x)) \in Z_{0}\right\}$ is null iff $\left\{x: q(x) B(x)^{-1} \in Z_{0}\right\}$ is null iff $\left\{x: q(x) \in Z_{0} \cdot B(x)\right\}$ is null iff $\left\{x: q(x) \in Z_{0}\right\}$ is null. Since $b$ is a homomorphism, $\left(p^{-1} \circ s^{-1}\right)\left(Z_{0}\right)$ is null. Since $h$ is a homomorphism and $s^{-1}\left(Z_{0}\right)$ is an invariant analytic subset of $Y, s^{-1}\left(Z_{0}\right)$ is null. Thus, $a$ is a homomorphism and $b$ is cohomologous to $a \circ h$.

3. SKEW PRODUCT ACTIONS. In this section, the Theorem is applied to study the relationship between conjugacy of skew product actions and similarity of groupoids. The following proposition is itself interesting and will be useful.

PROPOSITION 3.1 Let $h=(p, \phi): X \times G \rightarrow Y \times H$ be a homomorphism with Mackey dense range and suppose $J=(q, \psi): Y \times H \rightarrow Y \times H$ is a homomorphism such that $J \circ h \sim h$. Then $J \sim$ id where id $(y, h)=(y, h)$.

PROOF. Choose a Borel mapping $B=\left(B_{1}, B_{2}\right): X \rightarrow Y \times H$ satisfying

$$
\left(B_{1}(x), B_{2}(x)\right)(q(p(x)), \psi(p(x), \phi(x, g)))=(p(x), \phi(x, g))\left(B_{1}(x \cdot g), B_{2}(x \cdot g)\right)
$$

a.e. $(x, g)$. By Fubini's Theorem, equation (3.1) holds a.e. $x$ for a.e. $g$. But the set of all $g$ for which there exists a conull Borel subset $X_{g}$ of $X$, depending on $g$, so that (3.1) holds on $X_{g} \times\{g\}$ is a multiplicatively closed subset of $G$. Since $G$ is the only conull multiplicatively closed subset of $G$, then (3.1) holds a.e. $x$ for each $g$.

Consider the mapping $F: X \times_{\phi} H \times H \rightarrow H$ given by $F\left(x, h_{1}, h_{2}\right)=h_{2}^{-1} h_{1} B_{2}(x) \psi\left(p(x), h_{1}^{-1} h_{2}\right)$. Then $F\left(x \cdot g, h_{1} \phi(x, g), h_{2}\right)=h_{2}^{-1} h_{1} \phi(x, g) B_{2}(x \cdot g) \psi\left(p(x \cdot g), \phi(x, g)^{-1} h_{1}^{-1} h_{2}\right)=$ $h_{2}^{-1} h_{1} B_{2}(x) \psi(p(x), \phi(x, g)) \psi\left(p(x \cdot g), \phi(x, g)^{-1} h_{1}^{-1} h_{2}\right)=h_{2}^{-1} h_{1} B_{2}(x) \psi\left(p(x), h_{1}^{-1} h_{2}\right)=F\left(x, h_{1}, h_{2}\right)$ a.e. $x$, for each $g, h_{1}$, and $h_{2}$. Since $F$ is invariant in its first two variables and $(x, h) \rightarrow p(x) h^{-1}$ is an ergodic decomposition of $X \times_{\phi} H$, there exists a Borel mapping $C: Y \times H \rightarrow H$ for which

$$
C\left(p(x) h_{1}^{-1}, h_{2}\right)=h_{2}^{-1} h_{1} B_{2}(x) \psi\left(p(x), h_{1}^{-1} h_{2}\right) \quad \text { a.e. }\left(x, h_{1}, h_{2}\right) .
$$

Define $\eta: X \times H \rightarrow H$ by $\eta\left(x, h_{1}\right)=h_{1} B_{2}(x) \psi\left(p(x), h_{1}^{-1}\right)$. By using $(3.1), \eta\left(x \cdot g, h_{1} \phi(x, g)\right)=$ $h_{1} \phi(x, g) B_{2}(x \cdot g) \psi\left(p(x \cdot g), \phi(x, g)^{-1} h_{1}^{-1}\right)=h_{1} B_{2}(x) \psi(p(x), \phi(x, g)) \psi\left(p(x \cdot g), \phi(x, g)^{-1} h_{1}^{-1}\right)=$ $h_{1} B_{2}(x) \psi\left(p(x), h_{1}^{-1}\right)=\eta\left(x, h_{1}\right)$ a.e. $x$ for each $g, h_{1}$. Since $J$ has Mackey dense range, $(x, h) \mapsto p(x) h^{-1}$ is an ergodic decomposition of the the skew product $G$-space $X \times_{\phi} H$ and thus there exists a Borel mapping $\xi: Y \rightarrow H$ such that $\xi\left(p(x) h_{1}^{-1}\right)=h_{1} B_{2}(x) \psi\left(p(x), h_{1}^{-1}\right)$ a.e. $\left(x, h_{1}\right)$.

From (3.2), one finds $C\left(p(x) h_{1}^{-1}, h_{2}\right)=h_{2}^{-1} h_{1} B_{2}(x) \psi\left(p(x), h_{1}^{-1}\right) \psi\left(p(x) h_{1}^{-1}, h_{2}\right)=$ $h_{2}^{-1} \xi\left(p(x) h_{1}^{-1}\right) \psi\left(p(x) h_{1}^{-1}, h_{2}\right)$ a.e. $\left(x, h_{1}, h_{2}\right)$. Also, by the definition of $\xi$ and (3.2), one obtains $\xi\left(p(x) h_{1}^{-1} h_{2}\right)=h_{2}^{-1} h_{1} B_{2}(x) \psi\left(p(x), h_{1}^{-1} h_{2}\right)=C\left(p(x) h_{1}^{-1}, h_{2}\right)$ a.e. $\left(x, h_{1}, h_{2}\right)$. Consequently, $h_{2} \xi\left(p(x) h_{1}^{-1} h_{2}\right)=\xi\left(p(x) h_{1}^{-1}\right) \psi\left(p(x) h_{1}^{-1}, h_{2}\right)$ a.e. $\left(x, h_{1}, h_{2}\right)$. Thus,

$$
\xi(y) \psi\left(y, h_{2}\right)=h_{2} \xi\left(y \cdot h_{2}\right) \text { a.e. }\left(y, h_{2}\right) .
$$


On the other hand, as a consequence of (3.1) and the fact that $J$ is a homomorphism, one finds $p(x) h_{1}^{-1} \xi\left(p(x) h_{1}^{-1}\right)=p(x) h_{1}^{-1} h_{1} B_{2}(x) \psi\left(p(x), h_{1}^{-1}\right)=p(x) B_{2}(x) \psi\left(p(x), h_{1}^{-1}\right)=$ $B_{1}(x) B_{2}(x) \psi\left(p(x), h_{1}^{-1}\right)=q(p(x)) \psi\left(p(x), h_{1}^{-1}\right)=q\left(p(x) h_{1}^{-1}\right)$ a.e. $\left(x, h_{1}\right)$. Thus, $y \cdot \xi(y)=q(y)$ a.e. $y$ and by (3.3), $(y, \xi(y))\left(q(y), \psi\left(y, h_{2}\right)\right)=\left(y, h_{2}\right)\left(y \cdot h_{2}, \xi\left(y \cdot h_{2}\right)\right)$ a.e. $\left(y, h_{2}\right)$. Hence, $J \sim i d$.

COROLLARY 3.2 Let $\phi: X \times G \rightarrow H$ be a cocycle with Mackey dense range. Suppose $J$ is an endomorphism of $H$ such that $J \circ \phi \sim \phi$. Then $J$ is an inner conjugation.

PROPOSITION 3.3 Suppose $h=(p, \phi): X \times G \rightarrow Y \times H$ and $h^{\prime}=\left(p^{\prime}, \phi^{\prime}\right): X \times G \rightarrow Y^{\prime} \times H^{\prime}$ are homomorphisms with Mackey dense range. If the skew product $G$ spaces $X \times{ }_{\phi} H$ and $X \times \times_{\phi^{\prime}} H^{\prime}$ are conjugate extensions of $X$ then $Y \times H$ and $Y^{\prime} \times H^{\prime}$ are similar groupoids.

PROOF. If $X \times_{\phi} H$ and $X \times_{\phi^{\prime}} H^{\prime}$ are conjugate extensions of $X$ then there is a Borel mapping $\xi: X \times_{\phi} H \rightarrow H^{\prime}$ satisfying $\xi(x \cdot g, h \phi(x, g))=\xi(x, h) \cdot \phi^{\prime}(x, g)$ a.e. $(x, h)$ for each $g$. Let $\alpha: X \times_{\phi} H \rightarrow Y^{\prime}$ be the Borel mapping given by $\alpha(x, h)=p^{\prime}(x) \cdot \xi(x, h)^{-1}$. Then $\alpha$ is $G$-invariant since $\alpha((x, h) \cdot g)=p^{\prime}(x \cdot g) \xi(x \cdot g, h \phi(x, g))^{-1}=p^{\prime}(x \cdot g) \phi^{\prime}(x, g)^{-1} \xi(x, h)^{-1}=p^{\prime}(x) \xi(x, h)^{-1}=\alpha(x, h)$ a.e. $(x, h)$ for all g. Consequently, $(\alpha(x, h), \xi(x, h))\left(p^{\prime}(x), \phi^{\prime}(x, g)\right)=(\alpha((x, h) \cdot g), \xi((x, h) \cdot g))$ a.e. $(x, h)$ for all $g$ and so $h^{\prime} \mid X \times_{\phi} H \times G$ is trivial. Similarly, $h \mid X \times_{\phi^{\prime}} H^{\prime} \times G$ is trivial.

By the Theorem, there must exist homomorphisms $J$ and $J^{\prime}$ such that $J^{\prime} \circ h^{\prime} \sim h$ and $J \circ h \sim h^{\prime}$. Thus, $J^{\prime} \circ J \circ h \sim h$ and $J^{\prime} \circ J \sim i d$ follows by Proposition 3.1. Similarly, $J \circ J^{\prime} \sim i d$. This shows $Y \times H$ and $Y^{\prime} \times H^{\prime}$ are similar groupoids.

In Proposition 3.3, if $Y$ and $Y^{\prime}$ are points one obtains an interesting corollary that extends Theorem 1.3 in Fabec [1]. Also, if $X$ is a point, the problem stated in the introduction is answered.

COROLLARY 3.4 Suppose $\phi: X \times G \rightarrow H$ and $\psi: X \times G \rightarrow K$ are cocycles with Mackey dense range. Then the following are equivalent

(a) $\phi$ restricted to $X \times_{\psi} K \times G$ and $\psi$ restricted to $X \times_{\phi} H \times G$ are both trivial

(b) there is an isomorphism $J$ from $H$ onto $K$ such that $J \circ \phi$ is cohomologous to $\psi$

(c) the skew product actions of $G$ on $X \times_{\phi} H$ and on $X \times_{\psi} K$ define conjugate extensions of $X$.

PROOF. If (a) holds then by the Theorem, there are homomorphisms $J_{1}: H \rightarrow K$ and $J_{2}: K \rightarrow H$ such that $J_{1} \circ \phi \sim \psi$ and $J_{2} \circ \psi \sim \phi$. So $J_{1} \circ J_{2} \circ \psi \sim \psi$ and $J_{2} \circ J_{1} \circ \phi \sim \phi$.

By Corollary 3.2, $J_{1}$ must be an isomorphism and (b) is proved.

If (b) holds then there is a Borel map $A: X \rightarrow K$ satisfying $J(\phi(x, g))=A(x) \psi(x, g) A(x \cdot g)^{-1}$ a.e. $x$ for each $g$. Let $\xi: X \times_{\phi} H \rightarrow X \times_{\psi} K$ be the function given by $\xi(x, h)=(x, J(h) A(x))$. Then $\xi(x \cdot g, h \phi(x, g))=(x \cdot g, J(h) J \phi(x, g) A(x \cdot g))=(x \cdot g, J(h) A(x) \psi(x, g))=\xi(x, h) \cdot g$ a.e. $(x, h)$ for each $g$. Note, $\xi$ is a Borel isomorphism whose inverse is given by $\eta(x, k)=\left(x, J^{-1}\left(k A(x)^{-1}\right)\right)$. Moreover, a direct calculation shows $\xi_{*}\left(\mu \times m_{H}\right) \sim \mu \times m_{K}$ where $m_{H}$ and $m_{K}$ are Haar measures on $H$ and $K$, respectively. Thus, $X \times_{\phi} H$ and $X \times_{\psi} K$ are conjugate extensions of $X$.

Finally, in the proof of Proposition 3.3 it is shown (c) implies (a). 
ACKNOWLEDGEMENT. I wish to thank the referee for suggesting a number of remarks and modifications on an earlier version of this paper. Finally, the results found here rest on ideas generously shared by Professor Fabec and to whom I am indebted.

\section{REFERENCES}

1. FABEC, R.C., Cocycles, extensions of group actions, and bundle representations, J. Funct. Anal. 56 (1984), 79-98.

2. FABEC, R.C., Induced group actions, representations and fibered skew product extensions, Trans. Amer. Math. Soc. 301 (1987), 489-513.

3. MACKEY, G.W., Ergodic theory and virtual groups, Math. Ann. 166 (1966), 187-207.

4. RAMSAY, A., Virtual groups and group actions, Adv.in Math. 6 (1971), 253-322.

5. RAMSAY, A., Subobjects of virtual groups, Pacific J. Math. 87 (1980), 389-454. 


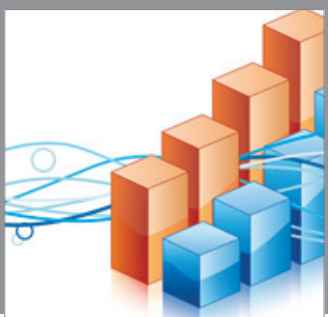

Advances in

Operations Research

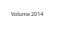

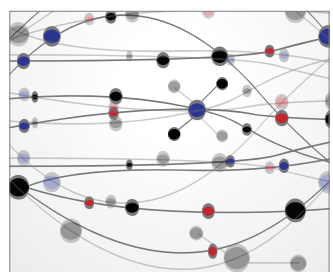

\section{The Scientific} World Journal
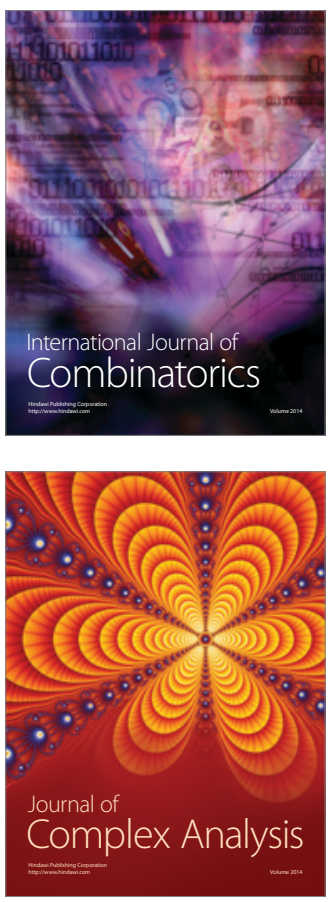

International Journal of

Mathematics and

Mathematical

Sciences
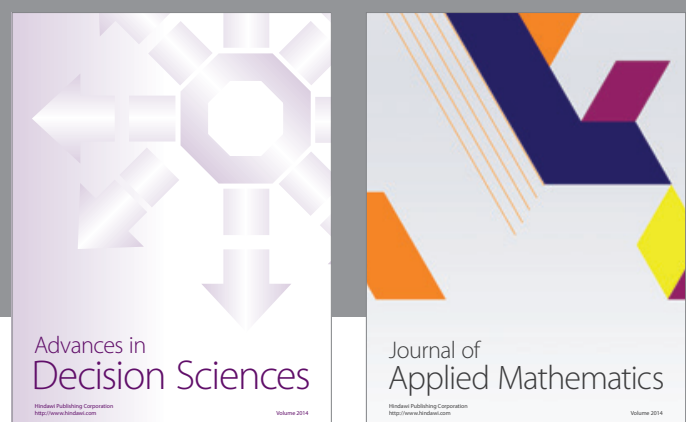

Journal of

Applied Mathematics
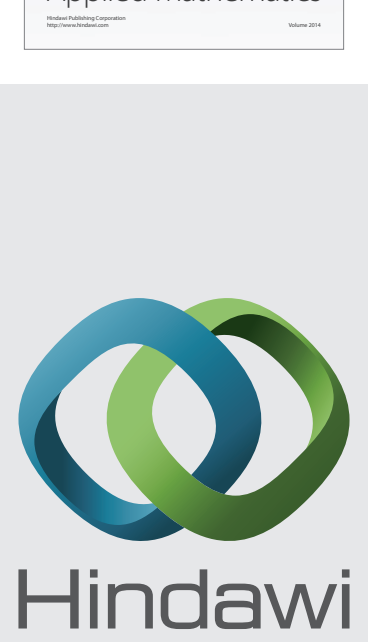

Submit your manuscripts at http://www.hindawi.com
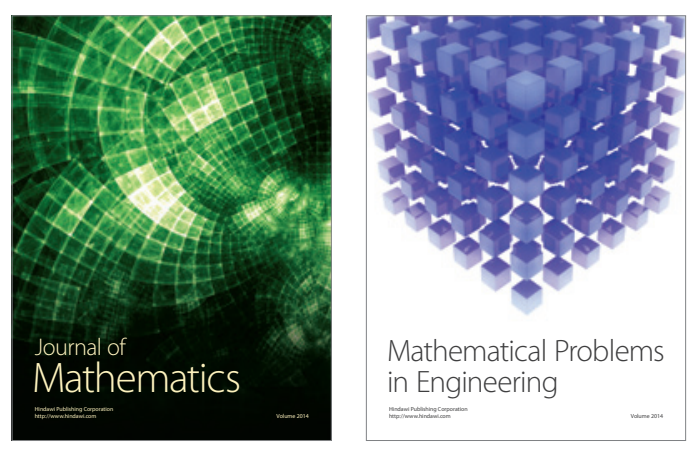

Mathematical Problems in Engineering
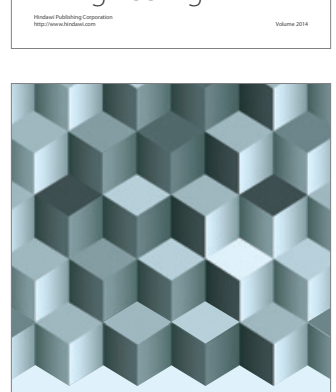

Journal of

Function Spaces
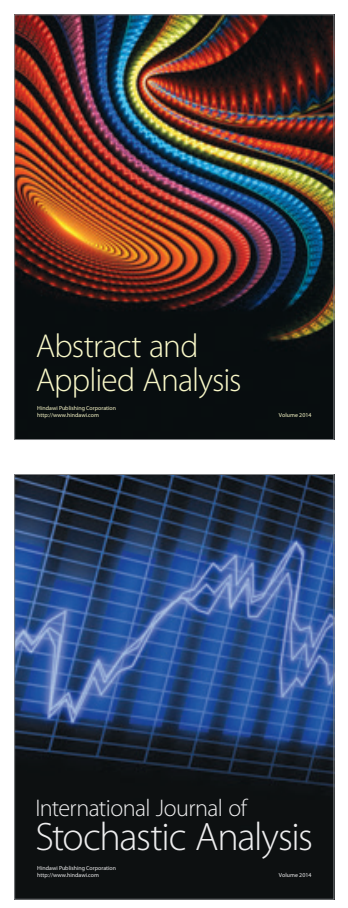

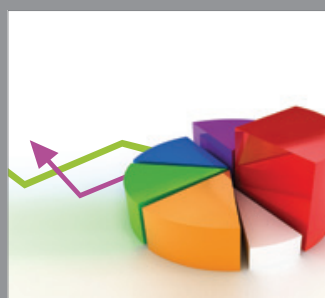

ournal of

Probability and Statistics

Promensencen
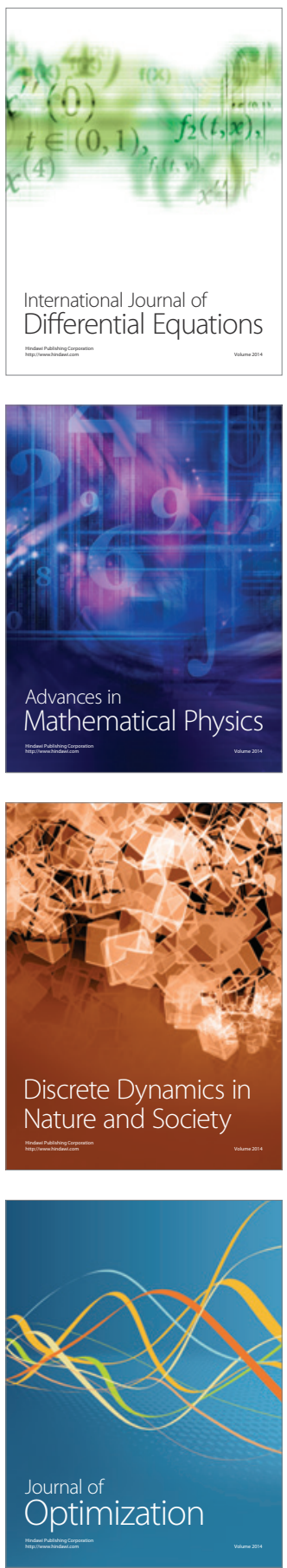